\title{
Eigenvalue inequalities for positive block matrices with the inradius of the numerical range.
}

\author{
Jean-Christophe Bourin* and Eun-Young Lee ${ }^{\dagger}$
}

\begin{abstract}
We prove the operator norm inequality, for a positive matrix partitioned into four blocks in $\mathbb{M}_{n}$,

$$
\left\|\left[\begin{array}{cc}
A & X \\
X^{*} & B
\end{array}\right]\right\|_{\infty} \leq\|A+B\|_{\infty}+\delta(X)
$$

where $\delta(X)$ is the diameter of the largest possible disc in the numerical range of $X$. This shows that the inradius $\varepsilon(X):=\delta(X) / 2$ satisfies $\varepsilon(X) \geq\|X\|_{\infty}-\left\|\left(\left|X^{*}\right|+|X|\right) / 2\right\|_{\infty}$. Several eigenvalue inequalities are derived. In particular, if $X$ is a normal matrix whose spectrum lies in a disc of radius $r$, the third eigenvalue of the full matrix is bounded by the second eigenvalue of the sum of the diagonal block,

$$
\lambda_{3}\left(\left[\begin{array}{cc}
A & X \\
X^{*} & B
\end{array}\right]\right) \leq \lambda_{2}(A+B)+r .
$$

We think that $r$ is optimal and we propose a conjecture related to a norm inequality of Hayashi. Keywords. Numerical range, Partitioned matrices, eigenvalue inequalities.

2010 mathematics subject classification. 15A60, 47A12, 15A42, 47A30.
\end{abstract}

\section{Introduction}

Positive matrices partitioned into four blocks play a central role in Matrix Analysis, and in applications, for instance quantum information theory. A lot of important theorems deal with these matrices. Some of these results give comparison between the full matrix and its diagonal blocks, in particular the sum of the diagonal blocks (the partial trace in the quantum terminology). This note focuses on a recent result of Bourin and Mhana [4], involving the numerical range of the offdiagonal block. Recall that a symmetric norm $\|\cdot\|$ on $\mathbb{M}_{2 n}$ means a unitarily invariant norm. It induces a symmetric norm on $\mathbb{M}_{n}$ in an obvious way. The Schatten $p$-norms $\|\cdot\|_{p}, 1 \leq p \leq \infty$, and the operator norm $(p=\infty)$ are classical examples of symmetric norms. The main result of [4] reads as follows.

*Funded by the ANR Projet (No. ANR-19-CE40-0002) and by the French Investissements d'Avenir program, project ISITE-BFC (contract ANR-15-IDEX-03).

${ }^{\dagger}$ This research was supported by Basic Science Research Program through the National Research Foundation of Korea (NRF) funded by the Ministry of Education (NRF-2018R1D1A3B07043682) 
Theorem 1.1. Let $\left[\begin{array}{cc}A & X \\ X^{*} & B\end{array}\right]$ be a positive matrix partitioned into four blocks in $\mathbb{M}_{n}$. Suppose that $W(X)$ has the width $\omega$. Then, for all symmetric norms,

$$
\left\|\left[\begin{array}{cc}
A & X \\
X^{*} & B
\end{array}\right]\right\| \leq\|A+B+\omega I\| .
$$

Here $I$ stands for the identity matrix, $W(X)$ denotes the numerical range of $X$, and the width of $W(X)$ is the smallest distance between two parallel straight lines such that the strip between these two lines contains $W(X)$. If $\omega=0$, that is $W(X)$ is a line segment, Theorem 1.1 was first proved by Mhanna [9]. Recently [3], Theorem 1.1 has been completed with the reversed inequality

$$
\left\|\left[\begin{array}{cc}
A & X \\
X^{*} & B
\end{array}\right]\right\| \geq\left\|\left[\begin{array}{cc}
\frac{A+B}{2}+d I & 0 \\
0 & \frac{A+B}{2}-d I
\end{array}\right]\right\| .
$$

where $d:=\min \{|z|: z \in W(X)\}$ is the distance from 0 to $W(X)$. Several applications were derived.

Some equality cases in Theorem 1.1 occur for the operator norm $\|\cdot\|_{\infty}$ with the following block matrices, where $a, b$ are two arbitrary nonnegative real numbers.

$$
\left[\begin{array}{ll}
\left(\begin{array}{ll}
a & 0 \\
0 & b
\end{array}\right) & \left(\begin{array}{ll}
0 & a \\
b & 0
\end{array}\right) \\
\left(\begin{array}{ll}
0 & b \\
a & 0
\end{array}\right) & \left(\begin{array}{ll}
b & 0 \\
0 & a
\end{array}\right)
\end{array}\right]
$$

This follows from the fact that $W\left(\left(\begin{array}{ll}0 & b \\ a & 0\end{array}\right)\right)$ has the width $2 \||a|-|b| \mid$, a consequence of the classical elliptical range theorem (see [8] for a short proof).

Though Theorem 1.1 is sharp for the operator norm, a subtle improvement is possible. This is our concern in the next section. Once again, a geometric feature of $W(X)$ will contribute: its inradius. Our approach leads to a remarkable list of eigenvalue that cannot be derived from the norm inequalities of Theorem 1.1. The last section is devoted to some related operator norm inequalities, in particular we will discuss a recent property due to Hayashi [6] and propose a conjecture.

\section{Eigenvalue inequalities}

We define the indiameter $\delta(\Lambda)$ of a compact convex set $\Lambda \subset \mathbb{C}$ as the diameter of the largest possible disc in $\Lambda$. For matrices $X \in \mathbb{M}_{n}$, we shorten $\delta(W(X))=: \delta(X)$. Recall that the numerical range of a two-by-two matrix is an elliptical disc (or a line segment, or a single point), see Li's short paper [8] or the famous book [7].

A matrix $X \in \mathbb{M}_{n}$ is identified as an operator on $\mathbb{C}^{n}$. If $\mathcal{S}$ is a subspace of $\mathbb{C}^{n}$, we denote by $X_{\mathcal{S}}$ the compression of $X$ onto $\mathcal{S}$. We then define the elliptical width of $X$ as

$$
\delta_{2}(X):=\sup _{\operatorname{dim} \mathcal{S}=2} \delta\left(X_{\mathcal{S}}\right) .
$$


Of course $\delta_{2}(X) \leq \delta(X) \leq \omega$ where $\omega$ still denotes the width of $W(X)$. If $X$ is a contraction, then $\delta_{2}(X) \leq 1$, while $\delta(X)$ may be arbitrarily close to 2 (letting $n$ be large enough). We state our main result.

Theorem 2.1. Let $\left[\begin{array}{cc}A & X \\ X^{*} & B\end{array}\right]$ be a positive matrix partitioned into four blocks in $\mathbb{M}_{n}$. Then, for all $j \in\{0,1, \ldots, n-1\}$,

$$
\lambda_{1+2 j}\left(\left[\begin{array}{cc}
A & X \\
X^{*} & B
\end{array}\right]\right) \leq \lambda_{1+j}(A+B)+\delta_{2}(X) .
$$

Here $\lambda_{1}(S) \geq \cdots \geq \lambda_{d}(S)$ stand for the eigenvalue of any Hermitian matrix $S \in \mathbb{M}_{d}$. If we denote by $\lambda_{1}^{\uparrow}(S) \leq \cdots \leq \lambda_{d}^{\uparrow}(S)$ these eigenvalues arranged in the increasing order, then Theorem 2.1 reads as

$$
\lambda_{2 k}^{\uparrow}\left(\left[\begin{array}{cc}
A & X \\
X^{*} & B
\end{array}\right]\right) \leq \lambda_{k}^{\uparrow}(A+B)+\delta_{2}(X) .
$$

for all $k \in\{1,2, \ldots, n\}$.

The case $j=0$ in Theorem 2.1 improves Theorem 1.1 for the operator norm. We may consider that Theorem 2.1 is trivial for $j=n-1$. Indeed, using the decomposition [2, Lemma 3.4],

$$
\left[\begin{array}{cc}
A & X \\
X^{*} & B
\end{array}\right]=U\left[\begin{array}{cc}
A & 0 \\
0 & 0
\end{array}\right] U^{*}+V\left[\begin{array}{cc}
0 & 0 \\
0 & B
\end{array}\right] V^{*}
$$

for some unitary matrices $U, V \in \mathbb{M}_{2 n}$, we obtain from Weyl's inequality [1, p. 62],

$$
\begin{aligned}
\lambda_{2 n-1}\left(\left[\begin{array}{cc}
A & X \\
X^{*} & B
\end{array}\right]\right) & \leq \lambda_{n}\left(\left[\begin{array}{cc}
A & 0 \\
0 & 0
\end{array}\right]\right)+\lambda_{n}\left(\left[\begin{array}{ll}
0 & 0 \\
0 & B
\end{array}\right]\right) \\
& =\lambda_{n}(A)+\lambda_{n}(B) \\
& \leq \lambda_{n}(A+B) .
\end{aligned}
$$

We turn to the proof of the theorem.

Proof. We first consider the case $j=0$. We may assume that the norm of the block matrix is strictly greater than the norms of its two diagonal blocks $A$ and $B$, otherwise the statement is trivial. Hence we have two nonzero (column) vectors $h_{1}, h_{2} \in \mathbb{C}^{n}$ such that $\left\|h_{1}\right\|^{2}+\left\|h_{2}\right\|^{2}=1$ and

$$
\lambda_{1}\left(\left[\begin{array}{cc}
A & X \\
X^{*} & B
\end{array}\right]\right)=\left\|\left[\begin{array}{cc}
A & X \\
X^{*} & B
\end{array}\right]\right\|_{\infty}=\left(\begin{array}{ll}
h_{1}^{*} & h_{2}^{*}
\end{array}\right)\left[\begin{array}{cc}
A & X \\
X^{*} & B
\end{array}\right]\left(\begin{array}{l}
h_{1} \\
h_{2}
\end{array}\right) .
$$

Therefore, denoting by $E_{1}$ and $E_{2}$ the rank one projections corresponding to the one dimensional subspaces spanned by $h_{1}$ and by $h_{2}$, we have

$$
\left\|\left[\begin{array}{cc}
A & X \\
X^{*} & B
\end{array}\right]\right\|_{\infty}=\left\|\left[\begin{array}{cc}
E_{1} & 0 \\
0 & E_{2}
\end{array}\right]\left[\begin{array}{cc}
A & X \\
X^{*} & B
\end{array}\right]\left[\begin{array}{cc}
E_{1} & 0 \\
0 & E_{2}
\end{array}\right]\right\|_{\infty}
$$


Hence, denoting by $F$ a rank two projection such that $E_{1} \leq F$ and $E_{2} \leq F$, we have

$$
\begin{aligned}
\left\|\left[\begin{array}{cc}
A & X \\
X^{*} & B
\end{array}\right]\right\|_{\infty} & =\left\|\left[\begin{array}{cc}
F & 0 \\
0 & F
\end{array}\right]\left[\begin{array}{cc}
A & X \\
X^{*} & B
\end{array}\right]\left[\begin{array}{cc}
F & 0 \\
0 & F
\end{array}\right]\right\|_{\infty} \\
& =\left\|\left[\begin{array}{cc}
F A F & F X F \\
F X^{*} F & F B F
\end{array}\right]\right\|_{\infty} .
\end{aligned}
$$

So, letting $\mathcal{S}$ denote the range of $F$, we have

$$
\left\|\left[\begin{array}{cc}
A & X \\
X^{*} & B
\end{array}\right]\right\|_{\infty}=\left\|\left[\begin{array}{cc}
A_{\mathcal{S}} & X_{\mathcal{S}} \\
X_{\mathcal{S}}^{*} & B_{\mathcal{S}}
\end{array}\right]\right\|_{\infty} .
$$

Hence applying Theorem 1.1 for the operator norm, we obtain

$$
\left\|\left[\begin{array}{cc}
A & X \\
X^{*} & B
\end{array}\right]\right\|_{\infty} \leq\left\|A_{\mathcal{S}}+B_{\mathcal{S}}\right\|_{\infty}+\varepsilon
$$

where $\varepsilon$ is the width of $W\left(X_{\mathcal{S}}\right)$. Since $W\left(X_{\mathcal{S}}\right)$ is an elliptical disc (as $X_{\mathcal{S}}$ acts on a two-dimensional space), its width equals to its indiameter, hence $\varepsilon \leq \delta_{2}(X)$, and since

$$
\left\|A_{\mathcal{S}}+B_{\mathcal{S}}\right\|_{\infty}=\left\|(A+B)_{\mathcal{S}}\right\|_{\infty} \leq\|A+B\|_{\infty}=\lambda_{1}(A+B)
$$

the proof for $j=0$ is complete.

We turn to the general case, $j=1, \ldots, n-1$. By the min-max principle,

$$
\begin{aligned}
\lambda_{1+2 j}\left(\left[\begin{array}{cc}
A & X \\
X^{*} & B
\end{array}\right]\right) & \leq \inf _{\operatorname{dim} \mathcal{S}=n-j} \lambda_{1}\left(\left[\begin{array}{cc}
A & X \\
X^{*} & B
\end{array}\right]_{\mathcal{S} \oplus \mathcal{S}}\right) \\
& =\inf _{\operatorname{dim} \mathcal{S}=n-j} \lambda_{1}\left(\left[\begin{array}{cc}
A_{\mathcal{S}} & X_{\mathcal{S}} \\
X_{\mathcal{S}}^{*} & B_{\mathcal{S}}
\end{array}\right]\right),
\end{aligned}
$$

hence, from the first part of the proof,

$$
\begin{aligned}
\lambda_{1+2 j}\left(\left[\begin{array}{cc}
A & X \\
X^{*} & B
\end{array}\right]\right) & \leq \inf _{\operatorname{dim} \mathcal{S}=n-j} \lambda_{1}\left(A_{\mathcal{S}}+B_{\mathcal{S}}\right)+\delta_{2}(X) \\
& =\lambda_{1+j}(A+B)+\delta_{2}(X)
\end{aligned}
$$

which is the desired claim.

If $X \in \mathbb{M}_{n}$, we denote by $\operatorname{dist}(X, \mathbb{C} I)$ the $\|\cdot\|_{\infty}$-distance from $X$ to $\mathbb{C} I$. Thus, for a scalar perturbation of a contraction, $X=\lambda I+C$ for some contraction $C \in \mathbb{M}_{n}$ and some $\lambda \in \mathbb{C}$, we have $\operatorname{dist}(X, \mathbb{C} I) \leq 1$.

Corollary 2.2. Let $\left[\begin{array}{cc}A & X \\ X^{*} & B\end{array}\right]$ be a positive matrix partitioned into four blocks in $\mathbb{M}_{n}$. Then, for all $j \in\{0,1, \ldots, n-1\}$,

$$
\lambda_{1+2 j}\left(\left[\begin{array}{cc}
A & X \\
X^{*} & B
\end{array}\right]\right) \leq \lambda_{1+j}(A+B)+\operatorname{dist}(X, \mathbb{C} I) .
$$


Proof. For any subspace $\mathcal{S} \subset C^{n}$, we have

$$
\operatorname{dist}(X, \mathbb{C} I) \geq \operatorname{dist}\left(X_{\mathcal{S}}, \mathbb{C} I_{\mathcal{S}}\right)
$$

If $\mathcal{S}$ has dimension 2 , then

$$
\operatorname{dist}\left(X_{\mathcal{S}}, \mathbb{C} I_{\mathcal{S}}\right) \geq \delta\left(W\left(X_{\mathcal{S}}\right)\right)
$$

Therefore $\operatorname{dist}(X, \mathbb{C} I) \geq \delta_{2}(X)$ and Theorem 2.1 completes the proof.

Corollary 2.3. Let $A, B \in \mathbb{M}_{n}$. Then, for every $j \geq 0$ such that $1+2 j \leq n$,

$$
\lambda_{1+2 j}\left(A^{*} A+B^{*} B\right) \leq \lambda_{1+j}\left(A A^{*}+B B^{*}\right)+\delta_{2}\left(A B^{*}\right)
$$

Proof. Note that

$$
\lambda_{1+2 j}\left(A^{*} A+B^{*} B\right)=\lambda_{1+2 j}\left(T^{*} T\right)=\lambda_{1+2 j}\left(T T^{*}\right)
$$

with $T=\left[\begin{array}{l}A \\ B\end{array}\right]$ and $T T^{*}=\left[\begin{array}{ll}A A^{*} & A B^{*} \\ B A^{*} & B B^{*}\end{array}\right]$ so that Theorem 2.1 yields the desired claim.

Corollary 2.4. Let $\left[\begin{array}{cc}A & N \\ N^{*} & B\end{array}\right]$ be a positive matrix partitioned into four blocks in $\mathbb{M}_{n}$. If $N$ is normal and its spectrum is contained in a disc of radius $r$, then,

$$
\lambda_{1+2 j}\left(\left[\begin{array}{cc}
A & N \\
N^{*} & B
\end{array}\right]\right) \leq \lambda_{1+j}(A+B)+r .
$$

for all $j=0,1, \ldots, n-1$.

Proof. Corollary 2.4 is a special case of corollary 2.2, as $N=\lambda I+R$, where $\lambda$ is the center of the disc of radius $r$ containing the spectrum of $N$, and $\|R\|_{\infty} \leq r$.

Question 2.5. Fix $r>0$ and $\varepsilon>0$. Can we find (with $n$ large enough) a normal matrix $N$ with spectrum in a disc of radius $r$ and a positive block matrix $\left[\begin{array}{cc}A & N \\ N^{*} & B\end{array}\right]$ such that

$$
\lambda_{1+2 j}\left(\left[\begin{array}{cc}
A & N \\
N^{*} & B
\end{array}\right]\right) \geq \lambda_{1+j}(A+B)+r-\varepsilon
$$

for some $j \in\{0, \ldots, n-1\}$ ? Is it true for for $j=0$ ? 


\section{Norm inequalities}

Corollary 2.4 with $j=0$ reads as follows.

Corollary 3.1. Let $\left[\begin{array}{cc}A & N \\ N^{*} & B\end{array}\right]$ be a positive matrix partitioned into four blocks in $\mathbb{M}_{n}$. If $N$ is normal and its spectrum is contained in a disc of radius $r$, then,

$$
\left\|\left[\begin{array}{cc}
A & N \\
N^{*} & B
\end{array}\right]\right\|_{\infty} \leq\|A+B\|_{\infty}+r .
$$

We do not know wether the constant $r$ is sharp or not (Question 2.5). If $n=2$, we can replace $r$ by 0 as the numerical range of $N$ is then a line segment. If $n=3$ there are some simple examples with $N=U$ unitary such that

$$
\left\|\left[\begin{array}{cc}
A & U \\
U^{*} & B
\end{array}\right]\right\|_{\infty}>\|A+B\|_{\infty} .
$$

See Hayashi's example in the discussion of [6, Problem 3] and the interesting study and examples in [5] where we further have $A+B=k I$ for some scalars $k$. The next result is due to Hayashi [6, Theorem 2.5].

Theorem 3.2. Suppose that $X \in \mathbb{M}_{n}$ is invertible with $n$ distinct singular values. If the inequality

$$
\left\|\left[\begin{array}{cc}
A & X \\
X^{*} & B
\end{array}\right]\right\|_{\infty} \leq\|A+B\|_{\infty}
$$

holds for all positive block-matrix with $X$ as off-diagonal block, then $X$ is normal.

Theorem 3.2 and Theorem 1.1 suggest a natural conjecture. If $W(T)$ is line segment, then $T$ is a so-called essentially Hermitian matrix.

Conjecture 3.3. Let $X \in \mathbb{M}_{n}$. If the inequality

$$
\left\|\left[\begin{array}{cc}
A & X \\
X^{*} & B
\end{array}\right]\right\|_{\infty} \leq\|A+B\|_{\infty}
$$

holds for all positive block-matrix with $X$ as off-diagonal block, then $X$ is essentially Hermitian.

If we replace the operator norm by the Frobenius (or Hilbert-Schmidt) norm $\|\cdot\|_{2}$ then the following characterization holds.

Proposition 3.4. Let $X \in \mathbb{M}_{n}$. Then, the inequality

$$
\left\|\left[\begin{array}{cc}
A & X \\
X^{*} & B
\end{array}\right]\right\|_{2} \leq\|A+B\|_{2}
$$

holds for all positive block-matrix with $X$ as off-diagonal block if and only if $X$ is normal. 
Proof. Suppose that $X$ is normal. To prove the inequality, squaring both side, it suffices to establish the trace inequality

$$
\operatorname{Tr} X^{*} X \leq \operatorname{Tr} A B
$$

Note that $X=A^{1 / 2} K B^{1 / 2}$, for some contraction $K$. Recall that, for all symmetric norms on $\mathbb{M}_{n}$, and any normal matrix $N \in \mathbb{M}_{n}$, decomposed as $N=S T$, we have $\|N\| \leq\|T S\|([1$, p. 253]). Therefore

$$
\|X\|=\left\|A^{1 / 2} K B^{1 / 2}\right\| \leq\left\|K B^{1 / 2} A^{1 / 2}\right\| .
$$

Squaring this inequality with the Frobenius norm yields the desired inequality (3.1).

Suppose that $X$ is nonnormal, and note that $\left[\begin{array}{cc}\left|X^{*}\right| & X \\ X^{*} & |X|\end{array}\right]$ is positive semidefinite and satisfies

$$
\left\|\left[\begin{array}{cc}
\left|X^{*}\right| & X \\
X^{*} & |X|
\end{array}\right]\right\|_{2}^{2}=4\||X|\|_{2}^{2}
$$

while

$$
\left\|\left|X^{*}\right|+|X|\right\|_{2}^{2}=2\||X|\|_{2}^{2}+2 \operatorname{Tr}|X|\left|X^{*}\right|
$$

In the Hilbert space $\left(\mathbb{M}_{n},\|\cdot\|_{2}\right)$, the assumption

$$
\||X|\|_{2}=\left\|\left|X^{*}\right|\right\|_{2}, \quad|X| \neq\left|X^{*}\right|
$$

ensures strict inequality in the Cauchy-Schwarz inequality

$$
\operatorname{Tr}|X|\left|X^{*}\right|<\|X\|_{2}^{2}
$$

Therefore

$$
\left\|\left|X^{*}\right|+|X|\right\|_{2}^{2}<\left\|\left[\begin{array}{cc}
\left|X^{*}\right| & X \\
X^{*} & |X|
\end{array}\right]\right\|_{2}^{2}
$$

and this completes the proof.

Proposition 3.4 suggests a question: for which $p \in[1, \infty]$, the schatten $p$-norm inequality

$$
\left\|\left[\begin{array}{cc}
A & N \\
N^{*} & B
\end{array}\right]\right\|_{p} \leq\|A+B\|_{p}
$$

holds for any positive partitioned matrices with a normal off-diagonal block $N$ ?

Corollary 3.5. Let $H, K, X \in \mathbb{M}_{n}$ be Hermitian. If $X$ is invertible and $H K$ is a scalar perturbation of a contraction, then,

$$
\left\|X H^{2} X+X^{-1} K^{2} X^{-1}\right\|_{\infty} \leq\left\|H X^{2} H+K X^{-2} K\right\|_{\infty}+1 .
$$


Proof. We apply Corollary 2.3 with $j=0$ and $A=H X, B=K X^{-1}$, to get

$$
\left\|X H^{2} X+X^{-1} K^{2} X^{-1}\right\|_{\infty} \leq\left\|H X^{2} H+K X^{-2} K\right\|_{\infty}+\delta_{2}(H K)
$$

Since $(H K)_{\mathcal{S}}$ is a scalar perturbation of a contraction acting on a space of dimension 2 , necessarily $\delta_{2}(H K) \leq 1$.

For a normal operator, the numerical range is the convex hull of the spectrum. For a non normal operator $X$, several lower bounds for the indiameter of $W(X)$ can be obtained from the left and right modulus $\left|X^{*}\right|$ and $|X|$.

Corollary 3.6. Let $X \in \mathbb{M}_{n}$ and let $f(t)$ and $g(t)$ are two nonnegative functions defined on $[0, \infty)$ such that $f(t) g(t)=t^{2}$ and $f(0)=g(0)=0$. Then,

$$
\delta_{2}(X) \geq\|f(|X|)+g(|X|)\|_{\infty}-\left\|f\left(\left|X^{*}\right|\right)+g(|X|)\right\|_{\infty} .
$$

Proof. First, observe that we have a function $h(t)$ defined on $[0, \infty)$ such that

$$
f(t)=t h^{2}\left(t^{1 / 2}\right), \quad g(t)=t h^{-2}\left(t^{1 / 2}\right),
$$

and $h(t)>0$ for all $t \geq 0$ (we may, for instance, set $h(0)=1$ ). Hence $h(T)$ is invertible for any positive $T$, and from the polar decomposition

$$
X=\left|X^{*}\right|^{1 / 2} U|X|^{1 / 2}
$$

with a unitary factor $U$, we infer the factorization

$$
X=\left|X^{*}\right|^{1 / 2} h\left(\left|X^{*}\right|^{1 / 2}\right) U|X|^{1 / 2} h^{-1}\left(|X|^{1 / 2}\right)
$$

Thus $X=A B^{*}$ where $A=\left|X^{*}\right|^{1 / 2} h\left(\left|X^{*}\right|^{1 / 2}\right)$ and $B^{*}=U|X|^{1 / 2} h^{-1}\left(|X|^{1 / 2}\right)$. Therefore Corollary 2.3 yields

$$
\left\|\left|X^{*}\right| h^{2}\left(\left|X^{*}\right|^{1 / 2}\right)+U|X| h^{-2}\left(|X|^{1 / 2}\right) U^{*}\right\|_{\infty} \leq\left\|\left|X^{*}\right| h^{2}\left(\left|X^{*}\right|^{1 / 2}\right)+|X| h^{-2}\left(|X|^{1 / 2}\right)\right\|_{\infty}+\delta_{2}(X)
$$

Using (3.2) and the fact that $\varphi\left(\left|X^{*}\right|\right)=U \varphi(|X|) U^{*}$ for any function $\varphi(t)$ defined on $[0, \infty)$, the proof is complete.

The following special case shows that Corollary 3.6 is rather optimal.

Corollary 3.7. If $X \in \mathbb{M}_{n}$ has a numerical range of inradius $\varepsilon(X)$, then, for all $a \in \mathbb{C}$,

$$
\varepsilon(X) \geq\|X-a I\|_{\infty}-\left\|\frac{|X-a I|+\left|X^{*}-\bar{a} I\right|}{2}\right\|_{\infty} .
$$

If $X \in \mathbb{M}_{2}$ and $a=\tau$ is the normalized trace of $X$, then this inequality is an equality. 
Proof. Applying Corollary 3.6 with $X-a I$ and $f(t)=g(t)=t$ yields the inequality. If $X \in \mathbb{M}_{2}$, then $X$ is unitarily equivalent to

$$
\left(\begin{array}{ll}
\tau & y \\
x & \tau
\end{array}\right)
$$

So

$$
\begin{aligned}
\|X-\tau I\|_{\infty}-\left\|\frac{|X-\tau I|+\left|X^{*}-\bar{\tau} I\right|}{2}\right\|_{\infty} & =\left\|\left(\begin{array}{cc}
|x| & 0 \\
0 & |y|
\end{array}\right)\right\|_{\infty}-\frac{1}{2}\left\|\left(\begin{array}{cc}
|x|+|y| & 0 \\
0 & |x|+|y|
\end{array}\right)\right\|_{\infty} \\
& =\frac{1}{2} \| x|-| y|| \\
& =\varepsilon(X)
\end{aligned}
$$

establishing the desired equality.

The special case $f(t)=g(t)=t$ in Corollary 3.6 seems important, we record it as a proposition:

Proposition 3.8. The elliptical width of the numerical range of $X \in \mathbb{M}_{n}$ satisfies

$$
\delta_{2}(X) \geq 2\|X\|_{\infty}-\left\||X|+\left|X^{*}\right|\right\|_{\infty}
$$

In particular, the inradius $\varepsilon(X)$ of the numerical range of $X$ satisfies

$$
\varepsilon(X) \geq\|X\|_{\infty}-\left\|\left(|X|+\left|X^{*}\right|\right) / 2\right\|_{\infty}
$$

Remark 3.9. Our results still hold for operators on infinite dimensional separable Hilbert space (assuming in Corollary 3.6 that $f(t)$ and $g(t)$ are Borel functions).

\section{References}

[1] R. Bhatia, Matrix Analysis, Gradutate Texts in Mathematics, Springer, New-York, 1996.

[2] J.-C. Bourin and E.-Y. Lee, Unitary orbits of Hermitian operators with convex or concave functions, Bull. Lond. Math. Soc. 44 (2012), no. 6, 1085-1102.

[3] J.-C. Bourin and E.-Y. Lee, Numerical range and positive block matrices. Bull. Aust. Math. Soc. 103 (2021), no. 1, 69-77.

[4] J.-C. Bourin and A. Mhanna, Positive block matrices and numerical ranges, C. R. Acad. Sci. Paris, 355 no. 10 (2017) 1077-1081.

[5] M. Gumus, J. Liu, S. Raouafi, T.-Y. Tam, Positive semi-definite $2 \times 2$ block matrices and norm inequalities, Linear Algebra Appl. 551 (2018) 83-91.

[6] T. Hayashi, On a norm inequality for a positive block-matrix, Linear Algebra Appl. 566 (2019), 86-97. 
[7] R.A. Horn, C.R. johnson, Topics in matrix analysis, Cambridge University Press, Cambridge, 1991.

[8] C.-K. Li, A simple proof of the elliptical range theorem, Proc. Amer. Math. Soc. 124 (1996), no. 7, 1985-1986.

[9] A. Mhanna, On symmetric norm inequalities and positive definite block-matrices, Math. Ineq. Appl., 21 (2018), no. 1, 133-138.

Eun-Young Lee

Department of mathematics, KNU-Center for Nonlinear Dynamics, Kyungpook National University,

Daegu 702-701, Korea.

Email: eylee89@knu.ac.kr

Jean-Christophe Bourin

Laboratoire de mathématiques, Université de Bourgogne Franche-Comté, 25000 Besançon, France.

Email: jcbourin@univ-fcomte.fr 